International Journal of Pure and Applied Mathematics

Volume 85 No. 1 2013, 83-93

ISSN: 1311-8080 (printed version); ISSN: 1314-3395 (on-line version)

url: http://www.ijpam.eu

doi: http://dx.doi.org/10.12732/ijpam.v85i1.7

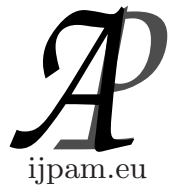

\title{
UNIT FRACTIONS THAT SUM TO 1
}

\author{
Yutaka Nishiyama
}

Department of Business Information

Faculty of Information Management

Osaka University of Economics

2, Osumi Higashiyodogawa Osaka, 533-8533, JAPAN

\begin{abstract}
This paper presents and solves a problem related to decomposing 1 into a sum of unit fractions. Decomposition into two terms, composition of two terms, decomposition into three terms, and other methods are used to find ways to maximize the number of unique terms, and a decomposition into 42 terms is demonstrated.
\end{abstract}

AMS Subject Classification: 11A02, 97F40, 00A08

Key Words: unit fraction, perfect number, hyperbola, integral, log function

\section{Starting with Perfect Numbers}

A fraction with 1 as its numerator is called a unit fraction, and 1 can be decomposed into a sum of unit fractions. A simple decomposition is as follows.

$$
1=\frac{1}{2}+\frac{1}{3}+\frac{1}{6}
$$

Multiplying both sides by 6 , we get

$$
6=3+2+1
$$

making it clear that the equation holds.

Received: December 21, 2012

(c) 2013 Academic Publications, Ltd. url: www.acadpubl.eu 
About 20 years ago, I began thinking about a certain problem related to unit fractions [1]. Limiting ourselves to two-digit denominators (in other words, natural numbers of 99 or less), what is the largest number of unit fraction terms that will sum up to 1 ?

A given natural number $m$ is called a perfect number when the sum of its divisors (including 1, but excluding $m$ ) equals itself. As an example, 6 is a perfect number, as is 28 (with divisors 14, 7, 4, 2, and 1):

$$
28=14+7+4+2+1 \text {. }
$$

Dividing both sides by 28 results in a decomposition of 1 into a sum of unit fractions with five terms:

$$
1=\frac{1}{2}+\frac{1}{4}+\frac{1}{7}+\frac{1}{14}+\frac{1}{28}
$$

There are many other perfect numbers $(496,8128,33550336$, and 8589869056, for example). While we could similarly decompose these numbers as above, the condition that each denominator must be 99 or less limits which ones we can use. For example 496 is out because two of its divisors have three digits:

$$
496=248+124+62+31+16+8+4+2+1 .
$$

By trial and error I managed to find a sum of 42 terms that met the conditions. I also learned that different methods of decomposition lead to different solutions. What I did not accomplish was a proof that 42 was the largest possible number of terms, so for all I know a 43-term solution awaits.

In this article, I would like to show how I found my 42-term solution. Please note that this is a fun problem to work on, so the motivated reader may wish to take a stab at it before reading on and then compare our solutions.

\section{Decomposition and Composition Into Two Terms}

Let's begin with the following 3-term solution:

$$
1=\frac{1}{2}+\frac{1}{3}+\frac{1}{6}
$$

Since we know that we will be dealing with unit fractions, we can save space by omitting the unit numerators, writing fractions like $\frac{1}{2}, \frac{1}{3}, \frac{1}{6}$ as $/ 2, / 3, / 6$. This allows us to rewrite the equation above as follows.

$$
1=/ 2+/ 3+/ 6 \text {. }
$$


From this, we can further decompose the initial $/ 2$ term for a total of 9 terms.

$$
\begin{aligned}
1 & =/ 2+/ 3+/ 6 \\
& =(/ 3+/ 6)+/ 3+/ 6 \\
& =(4 / 15+/ 15)+(/ 6)+/ 3+/ 6 \\
& =(4 / 15)+(/ 30+/ 30)+(/ 6)+/ 3+/ 6 \\
& =(/ 5+/ 15)+/ 30+(/ 45+/ 90)+(/ 9+/ 18)+/ 3+/ 6 \\
& =/ 3+/ 5+/ 6+/ 9+/ 15+/ 18+/ 30+/ 45+/ 90 .
\end{aligned}
$$

Next, we can use

$$
/ 3=/ 4+/ 12, \quad / 6=/ 7+/ 42
$$

to replace the $/ 3$ and $/ 6$, resulting in a total of 11 terms

$$
1=/ 4+/ 5+/ 7+/ 9+/ 12+/ 15+/ 18+/ 30+/ 42+/ 45+/ 90 .
$$

We can generalize our methods for decomposition and composition into five rules, as follows.

Rule 1: Decomposition Into Two Terms: Let's look at how a unit fraction $/ n$ can be decomposed into two differing unit fractions. Assume that the unit fraction's denominator can be factored as $n=a \times b$, where $a, b$ are allowed to be 1 . This allows for decomposition into two unit fractions, as follows

$$
\begin{aligned}
/ n= & /(a \times b)=(a+b) /((a \times b) \times(a+b)) \\
& =/(b \times(a+b))+/(a \times(a+b)) \\
& =/(a \times(a+b))+/(b \times(a+b))
\end{aligned}
$$

For example, for the unit fraction $/ 30$ we have $n=30$, which can be factored as $30=5 \times 6$. Then,

$$
\begin{gathered}
n=30, a=5, b=6, \\
a+b=11, \\
a \times(a+b)=55, b \times(a+b)=66 \\
/ 30=/ 55+/ 66
\end{gathered}
$$

We can repeat this to create as many decompositions as we like. Doing so to the $/ 3, / 6$ above results in the following. 


$$
\begin{aligned}
& / 3=/(1 \times 3)=/(1 \times(1+3))+/(3 \times(1+3))=/ 4+/ 12 \\
& / 6=/(1 \times 6)=/(1 \times(1+6))+/(6 \times(1+6))=/ 7+/ 42
\end{aligned}
$$

Doing this is relatively simple by hand, but you can also use spreadsheet software to speed things along.

We can do this again for the /42,/7 terms in Equation (3).

$$
\begin{gathered}
/ 42=/(6 \times 7)=/(6 \times(6+7))+/(7 \times(6+7))=/ 78+/ 91 \\
/ 7=/(1 \times 7)=/(1 \times(1+7))+/(7 \times(1+7))=/ 8+/ 56
\end{gathered}
$$

The following are some more applications of Rule 1.

$$
\begin{gathered}
/ 8=/ 9+/ 72 \\
/ 10=/ 14+/ 35 \\
/ 18=/ 22+/ 99 \\
/ 24=/ 33+/ 88 \\
/ 28=/ 44+/ 77 \\
/ 30=/ 55+/ 66 .
\end{gathered}
$$

Rule 2: Decomposition Into $n$ Terms by Multiplying the Denominator by $n$

Multiplying the denominator $a$ of $/ a$ by $n$ decomposes the unit fraction into $n$ unit fraction terms with $n \times a$ as a denominator

$$
/ a=/(n \times a)+/(n \times a)+\cdots+/(n \times a) .
$$

Using cases of $n=2$ or $n=3$, we get the following.

$$
\begin{aligned}
& / 4=/ 8+/ 8 \\
& / 5=/ 10+/ 10 \\
& / 8=/ 24+/ 24+/ 24 \\
& / 9=/ 18+/ 18 \\
& / 14=/ 28+/ 28
\end{aligned}
$$




$$
\begin{aligned}
& / 15=/ 30+/ 30 \\
& / 18=/ 36+/ 36 \\
& / 35=/ 70+/ 70
\end{aligned}
$$

Rule 3: Decomposition Into Three Terms: This rule performs a decomposition like this

$$
/ n=/ a+/ b+/ c
$$

but it is the most difficult to find. The method of calculation is described in the following section. Some examples of applying the rule are below

$$
\begin{aligned}
& / 9=/ 14+/ 35+/ 90 \\
& / 10=/ 17+/ 34+/ 85 \\
& / 12=/ 26+/ 39+/ 52 \\
& / 20=/ 38+/ 76+/ 95
\end{aligned}
$$

Rule 4: Transformation of a Decomposition Into Two Other Terms: After performing the standard decomposition into two terms, $/ a=/ b+/ c$, the denominator of each unit fraction can by multiplied by $n$ to create a decomposition into two new terms

$$
\begin{aligned}
/ a & =/ b+/ c \\
/(n \times a) & =/(n \times b)+/(n \times c) .
\end{aligned}
$$

For example:

$$
\begin{aligned}
/ 4 & =/ 5+/ 20 \\
/(2 \times 4) & =/(2 \times 5)+/(2 \times 20) \\
/ 8 & =/ 10+/ 40 .
\end{aligned}
$$

The following are some decompositions that follow this rule.

$$
\begin{aligned}
& / 10=/ 12+/ 60 \\
& / 12=/ 16+/ 48 \\
& / 14=/ 21+/ 42 \\
& / 15=/ 18+/ 90
\end{aligned}
$$




$$
\begin{aligned}
& / 16=/ 20+/ 80 \\
& / 18=/ 27+/ 54 \\
& / 24=/ 32+/ 96 \\
& / 30=/ 50+/ 75 \\
& / 36=/ 63+/ 84 .
\end{aligned}
$$

Rule 5: Reverse Application of Decomposition Into Two Terms (Composition of Two Terms): For example, given the decomposition into two terms

$$
/ 15=/ 18+/ 90
$$

we can swap the left and right sides to create a composition of two terms.

$$
/ 18+/ 90=/ 15
$$

The following are some compositions that follow this rule

$$
\begin{aligned}
& / 28+/ 70=/ 20 \\
& / 72+/ 24=/ 18
\end{aligned}
$$

\section{Decomposing Into Three Terms}

In Rule 3 of the previous section, I mentioned that a decomposition as follows is possible

$$
/ 12=/ 26+/ 39+/ 52
$$

Let us consider how we might decompose a unit fraction $1 / m$ into three terms. The key to doing so is finding three numbers $a, b, c$ such that their least common multiple is the denominator $m$. We then multiply the numerator and the denominator of the original unit fraction $1 / m$ by the sum of those three numbers, $a+b+c$

$$
\begin{aligned}
1 / m & =(a+b+c) /(m \times(a+b+c)) \\
& =a /(m \times(a+b+c))+b /(m \times(a+b+c))+c /(m \times(a+b+c)) .
\end{aligned}
$$


Here, since the least common multiple of $a, b, c$ is $m$, we know that each of $a, b, c$ will evenly divide $m$. In other words, $a / m, b / m, c / m$ are each unit fractions. When the denominators of each are multiplied by $(a+b+c)$, we will again have unit fractions.

Let's take $1 / 12$ as an example, and decompose it into three terms. Three numbers having 12 as their lowest common multiple are 3,4 , and 6 , the sum of which is $3+4+6=13$. We therefore want to multiply the denominator of the unit fraction $1 / 12$ by $(3+4+6)$

$$
\begin{aligned}
1 / 12 & =(3+4+6) /(12 \times(3+4+6)) \\
& =3 /(12 \times 13)+4 /(12 \times 13)+6 /(12 \times 13) \\
& =1 /(4 \times 13)+1 /(3 \times 13)+1 /(2 \times 13) \\
& =1 / 52+1 / 39+1 / 26 \\
& =1 / 26+1 / 39+1 / 52
\end{aligned}
$$

Another three numbers having 12 as their lowest common multiple are 2, 4 , and 6 . The sum in this case is $2+4+6=12$, so we proceed in the same manner

$$
\begin{aligned}
1 / 12 & =(2+4+6) /(12 \times(2+4+6)) \\
& =2 /(12 \times 12)+4 /(12 \times 12)+6 /(12 \times 12) \\
& =1 /(6 \times 12)+1 /(3 \times 12)+1 /(2 \times 12) \\
& =1 / 72+1 / 36+1 / 24 \\
& =1 / 24+1 / 36+1 / 72
\end{aligned}
$$

\section{A Maximum of 62 Terms}

As it turns out, repeated composition and decomposition of two and three terms to find increasingly long sums equal to 1 is an excellent way to kill time. But just how far can we take it? A bit of integral calculus allows one to predict that the maximum value must be 62 , as follows.

We wish to compare the sum of the unit fractions with an integral for the parabola $y=1 / x$. Taking $[1, n]$ as the limits of integration, the interval width of 
$x$ becomes $n-1$, so we can use rectangular approximation with $n-1$ rectangles to derive the following inequality

$$
\sum_{i=2}^{n} \frac{1}{i}<\int_{1}^{n} \frac{d x}{x}<\sum_{i=1}^{n-1} \frac{1}{i}
$$

Figures 1 and 2 show a graphical representation of this.

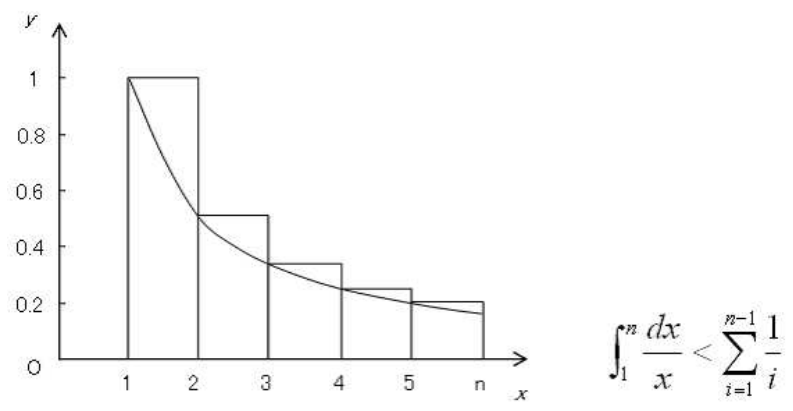

Figure 1

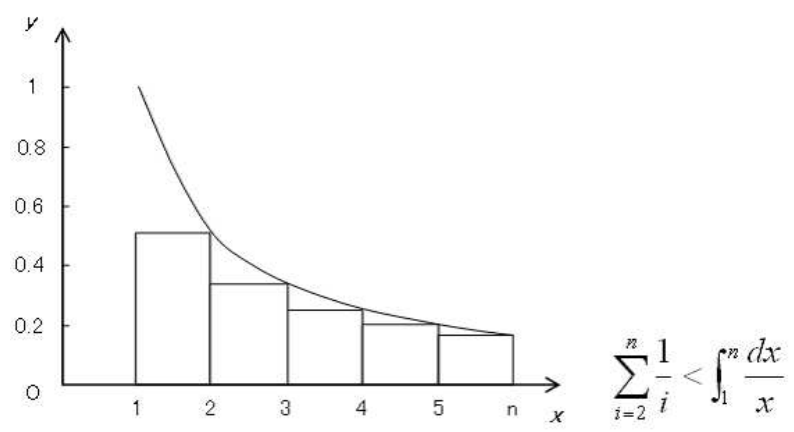

Figure 2

Some manipulation of the inequality gives

$$
\int_{1}^{n} \frac{d x}{x}+\frac{1}{n}<\sum_{i=1}^{n} \frac{1}{i}<\int_{1}^{n} \frac{d x}{x}+1
$$


and since the integral for $y=1 / x$ is $\log x$, we get

$$
\log n+\frac{1}{n}<\sum_{i=1}^{n} \frac{1}{n}<\log n+1
$$

The larger the denominator in a unit fraction the more terms we can add, so we should start with 99 and work our way down. In other words, we will begin with $\sum_{i=n}^{99} \frac{1}{i}$, and look for values of $n$ for which the sum does not exceed 1. Changing the limits of integration for the original inequality from $[1, n]$ to $[n, 99]$ we get

$$
\sum_{i=n+1}^{99} \frac{1}{i}<\int_{n}^{99} \frac{d x}{x}<\sum_{i=n}^{98} \frac{1}{i}
$$

which can be rewritten as

$$
\int_{n}^{99} \frac{d x}{x}+\frac{1}{99}<\sum_{i=n}^{99} \frac{1}{i}<\int_{n}^{99} \frac{d x}{x}+\frac{1}{n},
$$

the definite integral of which is

$$
\log 99-\log n+\frac{1}{99}<\sum_{i=n}^{99} \frac{1}{i}<\log 99-\log n+\frac{1}{n} .
$$

The inequality on the right says that in order for the sum of the unit fractions to not exceed 1 we must have

$$
\log 99-\log n+\frac{1}{n}<1
$$

Solving for $n$, we get

$$
n=38 .
$$

This indicates a sum from $1 / 38$ to $1 / 99$, so the number of terms is $99-$ $38+1=62$. I have found a solution with 42 terms, so a maximum of 62 sounds reasonable. 


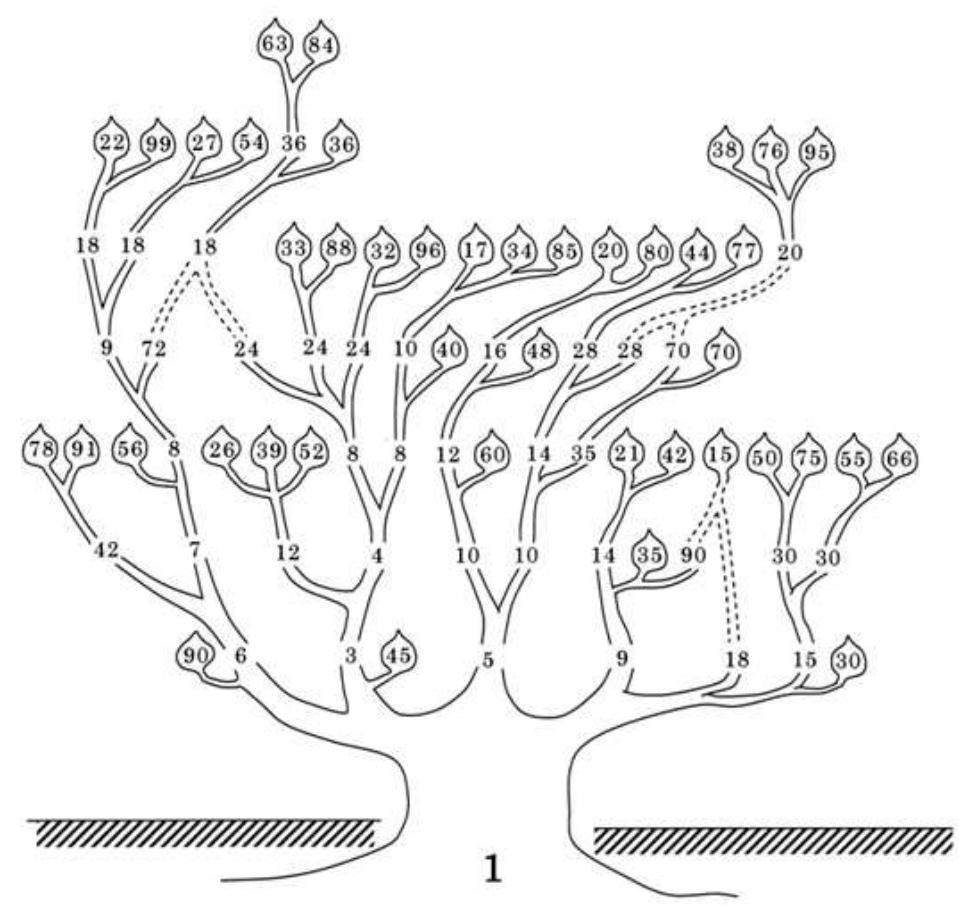

Figure 3: Unit fractions as a tree diagram

\section{A Tree Diagram}

My current solution with 42 different unit fraction terms looks like this.

$$
\begin{gathered}
1=/ 15+/ 17+/ 20+/ 21+/ 22+/ 26+/ 27+/ 30+/ 32+/ 33 \\
+/ 34+/ 35+/ 36+/ 38+/ 39+/ 40+/ 42+/ 44+/ 45+/ 48 \\
+/ 50+/ 52+/ 54+/ 55+/ 56+/ 60+/ 63+/ 66+/ 70+/ 75 \\
+/ 76+/ 77+/ 78+/ 80+/ 84+/ 85+/ 88+/ 90+/ 91+/ 95 \\
+/ 96+/ 99 .
\end{gathered}
$$

The replacement

$$
/ 15+/ 30+/ 90=/ 18+/ 24+/ 72
$$

can be made in this solution, giving an alternative 42-term answer.

$$
1=/ 17+/ 18+/ 20+/ 21+/ 22+/ 24+/ 26+/ 27+/ 32+/ 33
$$




$$
\begin{gathered}
+/ 34+/ 35+/ 36+/ 38+/ 39+/ 40+/ 42+/ 44+/ 45+/ 48 \\
+/ 50+/ 52+/ 54+/ 55+/ 56+/ 60+/ 63+/ 66+/ 70+/ 72 \\
+/ 75+/ 76+/ 77+/ 78+/ 80+/ 84+/ 85+/ 88+/ 91+/ 95 \\
+/ 96+/ 99 .
\end{gathered}
$$

Figure 3 shows solution (4) as a tree diagram, in which the trunk is our original value of 1 and the branches show the process of decomposition into two and three terms. Each of the 42 leaves is a 42 unique unit fraction term in the solution. I enjoy graphs like this, but a botanist might be disturbed by the floating branches (connected by dotted lines) that resulted from the three compositions that I performed

$$
\begin{aligned}
& / 90+/ 18=/ 15 \\
& / 72+/ 24=/ 18 \\
& / 28+/ 70=/ 20
\end{aligned}
$$

Perhaps a bit more searching would repair those places by removing the need for the compositionsc

\section{References}

[1] Y. Nishiyama, In search of an elegant solution, Mathematics Seminar, 31, No. 11 (1992), 106-107, In Japanese. 
